\title{
ESTUDO DE INCORPORAÇÃO DE NANOCARGAS DE SIC À MATRIZ DE POLI(METACRILATO DE METILA) VIA POLIMERIZAÇÃO EM MASSA*
}

\section{Resumo}

Flávio James Tommasini ${ }^{1}$ Leonardo da Cunha Ferreira ${ }^{2}$ Sergio Neves Monteiro Matheus $R$ Affonso ${ }^{4}$ Victor S Brandão $0^{5}$ Caio V C Lira ${ }^{6}$ Pedro H L M Lopes

A pesquisa foi realizada com o intuito de produzir nanocompósitos baseados em uma matriz polimérica de poli (metacrilato de metila) (PMMA) com nanocargas de carbeto de silício ( $\mathrm{SiC}$ ) dispersas na matriz orgânica. Os nanocompósitos foram produzidos através da polimerização em massa, a qual o peróxido de benzoíla foi utilizado como o iniciador. Os novos materiais foram produzidos sob a forma de filmes e consequentemente eles foram submetidos e caracterizados pelas análises de termogravimetria (TGA), espectroscopia de infravermelho por transformada Fourier (FTIR), fluorescência de raio-x (XRF), espectroscopia por energia dispersiva de raio-x (EDS) e microscopia eletrônica de varredura (FEGSEM). Os resultados obtidos durante a caracterização dos filmes dos nanocompósitos demonstraram a boa eficiência dos processos de sínteses e misturas das cargas de SiC na matriz orgânica.

Palavras-chave: Nanocompósitos; Carbeto de Silício; Poli (metacrilato de metila); Polimerização em Massa; Filmes Planos.

\section{STUDY ABOUT THE INCORPORATION OF SIC ONTO THE POLY (METIL METACRILATE) MATRIX FOR MASS POLYMERIZATON}

\section{Abstract}

Research was developed to production of nanocomposites materials, based on polymer matrix of poly (methyl methacrylate) (PMMA), containing the disperse nanoparticles of silicon carbide (SiC) into the organic matrix. The nanocomposites were produced by radical mass polymerization, which the benzoyl peroxide was used as initiator agent of the polymerization reaction. New materials were submitted and characterized by thermogravimetry (TGA), Fourier transform infrared spectroscopy (FTIR), X-ray fluorescence (XRF), X-ray dispersive energy spectroscopy (EDS) and scanning electron microscopy (FEGSEM). The obtained results, during the characterization of the nanocomposites flat films showed the efficiency of de synthesis and mixture of $\mathrm{SiC}$ fillers into the organic matrix.

Keywords: Nanocomposites; Silicon carbide; Poly (methyl methacrylate); Mass Polymerization; Polymer Films.

1 Desenho Industrial- Projeto de Produto, pós doutor, pesquisador, Departamento de Engenharia de Materiais, Instituto Militar de Engenharia, Rio de Janeiro- RJ, Brasil.

2 Químico, pós-doutor, pesquisador, Ciências e Tecnologia de Polímeros, Instituto de Macromoléculas Professora Eloisa Mano, Rio de Janeiro-RJ, e Brasil.

3 Engenharia de Materiais, Phd, professor, Instituto Militar de Engenharia, Rio de Janeiro-RJ, Brasil.

4 Engenharia de Materiais, graduando, iniciação científica, Departamento de Engenharia de Mecânica e Materiais, Instituto Militar de Engenharia, Rio de Janeiro-RJ, Brasil. 
5 Engenharia de Materiais, graduando, iniciação científica, Departamento de Engenharia de Mecânica e Materiais, Instituto Militar de Engenharia, Rio de Janeiro-RJ, Brasil.

6 Engenharia de Materiais, graduando, iniciação científica, Departamento de Engenharia de Mecânica e Materiais, Instituto Militar de Engenharia, Rio de Janeiro-RJ, Brasil.

7 Engenharia de Materiais, graduando, iniciação científica, Departamento de Engenharia de Mecânica e Materiais, Instituto Militar de Engenharia, Rio de Janeiro-RJ, Brasil.. 


\section{INTRODUÇÃO}

Atualmente, a diversidade de necessidades criadas pela sociedade por materiais mais funcionais, com melhor desempenho e propriedades diferenciadas tem focado maiores estudos para desenvolvimento de materiais e produtos inovadores. A elevada demanda por materiais com propriedades diferenciadas como resistência mecânica, características térmicas, elétricas, magnéticas, leveza, dureza tem sido o grande atrativo para o elevado desenvolvimento em pesquisas para novas ligas, compostos, compósitos, nanocompósitos, entre outros produtos avançados, geralmente com custos mais baixos e processabilidade versátil [1].

Entre os materiais muitos estudados estão os nanocompósitos, ou seja, materiais híbridos em que pelo menos um dos componentes possui suas dimensões nanométricas, e assim como os compósitos tradicionais, um dos materiais é a matriz e o segundo material se dispondo como fase dispersa [2]. Muitos estudos diversificando a matriz para nanocompósitos com propriedades determinadas e diferentes tipos de cargas (natureza orgânica ou inorgânica) têm sido realizadas por muitos pesquisadores pelo mundo [3]. A utilização de cargas com diferentes dimensões promove a obtenção de nanocompósitos com propriedades e aplicações diferenciadas [4]. Na atualidade, a produção, o processamento e modificação das características e propriedades dos polímeros são muito importantes e necessários para a indústria de polímero. As áreas de aplicação desses materiais poliméricos são as mais abrangentes, como eletrônica, medicina, transporte, construção, aviação, na produção de bandagens, colas, fitas, tintas, anteparos antibalísticos (coletes, fuselagem, materiais contra detecção de radares), entre outros [5].

Entre as várias rotas para a produção de nanocompósitos que são estudadas, as vias polimerização in situ são particularmente as mais interessantes. Essas técnicas possibilitam a distribuição mais homogênea das cargas inorgânicas em meio aos diversos tipos de matrizes orgânicas [6]. Um tipo de matriz polimérica muito utilizada tem sido as matrizes de poli (metacrilato de metila) (PMMA) não somente pelo baixo custo, como pelas suas propriedades termoplásticas. Entre as algumas características físicas e químicas cuja aplicação os tornam muito rentáveis estão a alta transparência adquirida, resistência térmica, boa processabilidade e versatilidade para aplicações.

Entre algumas nanocargas que têm aumentado os interesses para pesquisas está o carbeto de silício $(\mathrm{SiC})$. O alto interesse é devido ao desenvolvimento de cerâmica de alto desempenho, com baixo coeficiente de expansão térmica, alta condutividade térmica e alta resistência mecânica[7].

A pesquisa da síntese de nanocompósitos com matriz polimérica de poli (metacrilato de metila) contendo nanopartículas de carbeto de silício dispersas pode auxiliar na avaliação das interações e modificações possíveis com esse tipo de carga, sob dimensões nanométricas, para produção de materiais nanocompósitos de alto desempenho térmico, ótico, mecânico, químico, entres outras propriedades adquiridas pela matriz sintetizada de poli (metacrilato de metila).

\section{MATERIAIS E MÉTODOS}

O monômero de metacrilato de metila foi adquirido pela VETEC e previamente destilado com a presença de hidroquinona produzido pela ALDRICH. O processo de destilação foi realizado com um equipamento de destilação simples. O peróxido de benzoíla produzido pela ALDRICH foi utilizado como iniciador da reação de 
polimerização em massa. Para a síntese do nanocompósito foram utilizadas placas de petri como suporte e molde dos filmes, o agitador magnético com velocidades de agitação constante da reação no interior dos erlenmayers, sob aquecimento em meio de banho de silicone. Uma estufa regulada à $40^{\circ} \mathrm{C}$ foi utilizada para completar a polimerização.

\section{1- SÍNTESE DE POLI (METACRILATO DE METILA) E NANOCOMPÓSITOS CONTENDO SIC}

A partir de um erlenmayer seco contendo uma barra de agitação magnética foi introduzido o monômero de metacrilato de metila destilado com a hidroquinona e 0,20 g de peróxido de benzoíla como iniciador da reação. O recipiente foi imerso em banho de silicone na faixa de 80 e $90^{\circ} \mathrm{C}$, sob agitação constante para homogeneizar a mistura e impedir o aparecimento de bolhas de ar, retidas na matriz durante a reação de polimerização. $O$ aquecimento prosseguiu até determinada viscosidade, a qual o polímero em formação tornou-se perceptível. A reação final foi realizada em estufa à $40^{\circ} \mathrm{C}$, durante 24 horas.

A rota de síntese igual ao do PMMA puro com a adição das nanopartículas de SiC foi realizada utilizando as concentrações em massa $(\mathrm{m} \backslash \mathrm{m})$ de $0,1 \%, 0,25 \%, 0,5 \%$ e $1 \%$ de nanocargas de carbeto de silício. As nanopartículas foram adicionadas ao monômero antes da adição do iniciador (peróxido de benzoíla) no meio reacional.

Espectroscopia de energia dispersiva de raios-X (EDX): Esta análise foi realizada com um espectrômetro Shimadzu EDX 720, operando com feixe de elétrons com uma energia entre 20 e 40 kV sob vácuo. Cerca de 1 grama de amostra foi inserido no suporte de amostras e depois analisado.

\section{2- MÉTODOS DE CARACTERIZAÇÃO DOS MATERIAIS PUROS E NANOCOMPÓSITOS}

\subsubsection{Espectroscopia de energia dispersiva de raios-X (EDX)}

A análise foi realizada em um espectrômetro Shimadzu EDX 720, com feixe de elétrons, energia na faixa de 20 - $40 \mathrm{kV}$, sob vácuo. Cada amostra de material (cerca de 1 grama) foi inserida no suporte de amostras para análise e detecção.

\subsubsection{Espectroscopia por dispersão de energia (EDS)}

Esta técnica foi utilizada para detecção de elementos inorgânicos nas amostras dos 4 tipos de nanocompósitos produzidos. O detector utilizado foi o modelo QUANTAX 70 , conectado ao microscópio eletrônico modelo TM 3030, produzido pela Hitachi High Technologies.

\subsubsection{Análise termogravimétrica (TGA)}

As estabilidades térmicas do polímero e dos nanocompósitos foram analisadas pela análise de TGA. O equipamento utilizado é da marca TA Instruments, modelo Q500, empregando a taxa de velocidade de aquecimento de $10{ }^{\circ} \mathrm{C} / \mathrm{min}^{-1}$, com varredura nas faixas de temperaturas de 30 a $700^{\circ} \mathrm{C}$, sob uma atmosfera inerte de $\mathrm{N}_{2}$. Os 
resultados obtidos representaram os valores de temperatura inicial de degradação ( $\left.T_{\text {onset}}\right)$, temperatura máxima de degradação $\left(T_{\max }\right)$ e o resíduo após a temperatura de varredura final em $700^{\circ} \mathrm{C}$.

\subsubsection{Espectroscopia no Infravermelho de transformada de Fourier (FTIR)}

Esta técnica foi utilizada para caracterização da estrutura química dos materiais (polímero e carbeto de silício) e identificação dos grupos funcionais das nanocargas de carbeto de silício presentes nos nanocompósitos. Os espectros foram obtidos com um aparelho Perkin Elmer 100, no intervalo de comprimento de onda de 4000$500 \mathrm{~cm}^{-1}$.

\subsubsection{Microscopia eletrônica de varredura (MEV)}

A observação e análise da morfologia, microestrutura, interação e dispersão dos materiais e nanocompósitos foi realizada a partir de um microscópio eletrônico de varredura da marca FEI, tipo FEGSEM, modelo FEI Quanta 250. As imagens foram obtidas a partir do detector de elétrons secundários, sob alto vácuo e corrente variável, com a intensidade de feixe entre a faixa de 1 e $2 \mathrm{Kv}$. As amostras foram recobertas com platina $(\mathrm{Pt})$ para melhor resolução das imagens e evitar a degradação dos filmes das amostras produzidas. O detector para análise de espectroscopia de energia dispersiva (EDS) foi Bruker e acoplado ao microscópio eletrônico de varredura para verificação e semiquantificação composicional dos elementos das amostras.

\section{RESULTADOS E DISCUSSÃO}

A análise por EDX forneceu as quantidades de elementos inorgânicos presentes nos nanocompósitos sintetizados (a Tabela 1). Conforme esperado, a presença de silício foi detectada e o aumento da quantidade do elemento silício foi elevada com o aumento de SiC, adicionado ao monômero para a reação de polimerização.

Tabela 1: Resultados de EDX para os nanocompósitos

\begin{tabular}{lcc}
\hline Nanocompósitos (\%) & $\begin{array}{c}\text { \% de Silício } \\
(\mathbf{m} \backslash \mathbf{m})\end{array}$ & $\begin{array}{c}\text { \% de Cloro } \\
(\mathbf{m} \backslash \mathbf{m})\end{array}$ \\
\hline PMMAISiC 0,1 & 30 & 15 \\
\hline PMMAISiC 0,25 & 75 & 13 \\
\hline PMMAISiC 0,5 & 67 & 9 \\
\hline PMMAISiC 1 & 81 & 4 \\
\hline
\end{tabular}

A análise de espectroscopia de infravermelho por transformada Fourier (FTIR) sugeriu o sucesso na obtenção da matriz polimérica de PMMA e a incorporação das partículas de $\mathrm{SiC}$ nas matrizes dos nanocompósitos produzidos. Segundo a Figura 1 , a respeito dos espectros do polímero puro sintetizado e dos nanocompósitos contendo diferentes porcentagens de nanocargas de SiC, foi possível a observação das bandas de absorção referentes ao PMMA e alguns indícios de absorção referentes às partículas de SiC. Em todas as amostras foi observada a presença da banda de absorção localizada em $1723 \mathrm{~cm}^{-1}$, associada ao estiramento do grupo carbonila $(\mathrm{C}=\mathrm{O})$ do polímero [7]. Há absorções localizadas entre 2995 e $2951 \mathrm{~cm}^{-1}$ (referentes aos estiramentos $\mathrm{C}-\mathrm{H}$ dos grupos $-\mathrm{CH} 3$ e $-\mathrm{CH} 2$, respectivamente), em $1062 \mathrm{~cm}^{-1}$ (referente ao estiramento da ligação C-O). As bandas localizadas em 
1388 e $754 \mathrm{~cm}^{-1}$ podem ser atribuídas às vibrações do grupo $\alpha$-metila e a em 987 $\mathrm{cm}^{-1}$ é a vibração característica de absorção de PMMA, juntamente com as bandas em $1062 \mathrm{~cm}^{-1}$ e $843 \mathrm{~cm}^{-1}$. As principais bandas de absorção do SiC foram observadas entre as bandas de 900 e $500 \mathrm{~cm}^{-1}$ e são referentes ao estiramento da ligação Si-C [6]. Nesse intervalo foram verificadas a presença de bandas que não são observadas no espectro da matriz polimérica pura. $O$ fato de não se observar variações significativas de energias de absorção entre os espectros de PMMA puro e dos nanocompósitos pode estar ligado a uma fraca interação entre a nanocarga e a matriz.

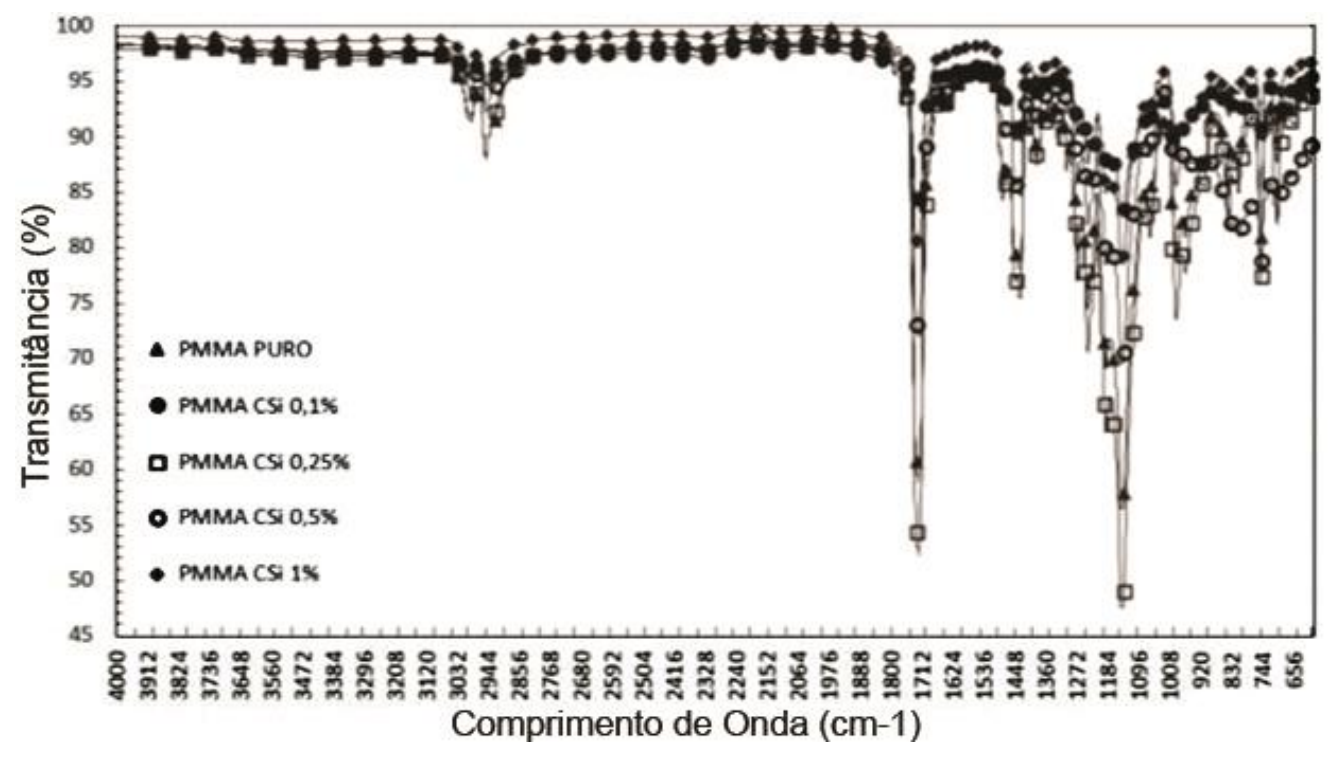

Figura 1: Comparação entre os espectros de FTIR da matriz pura sintetizada e dos seus respectivos nanocompósitos.

Os resultados obtidos pelas análises de TGA dos respectivos nanocompósitos mostram alguma evidência de sucesso da síntese da matriz polimérica pura e com a presença das diferentes proporções de nanocargas de carbeto de silício. Enquanto que para cada curva de nanocompósito produzido foram observados três estágios de perda de massa, como pode ser visualizado através da Figura 2. Segundo Araújo e colaboradores, a degradação em três etapas do PMMA é típica daquele produzido via mecanismo radicalar [8]. A primeira etapa é referente a cisão das fracas ligações entre peróxido e hidroperóxido, possivelmente devido à combinação de monômeros com $\mathrm{O}_{2}$ durante a síntese. A segunda etapa pode ser resultado da cisão de terminações insaturadas existentes, enquanto a terceira etapa se deve a maior parte pela quebra da cadeia principal de PMMA [9]. Através da análise foi possível supor que o iniciador ainda existente nas amostras exerce influência no comportamento das curvas dos respectivos materiais.

Através da análise de TGA foi possível conjecturar que o acréscimo de $\mathrm{SiC}$ reduziu a estabilidade térmica da matriz, como visto pelas derivadas termogravimétricas e resíduo final das respectivas degradações (Tabela 2). 


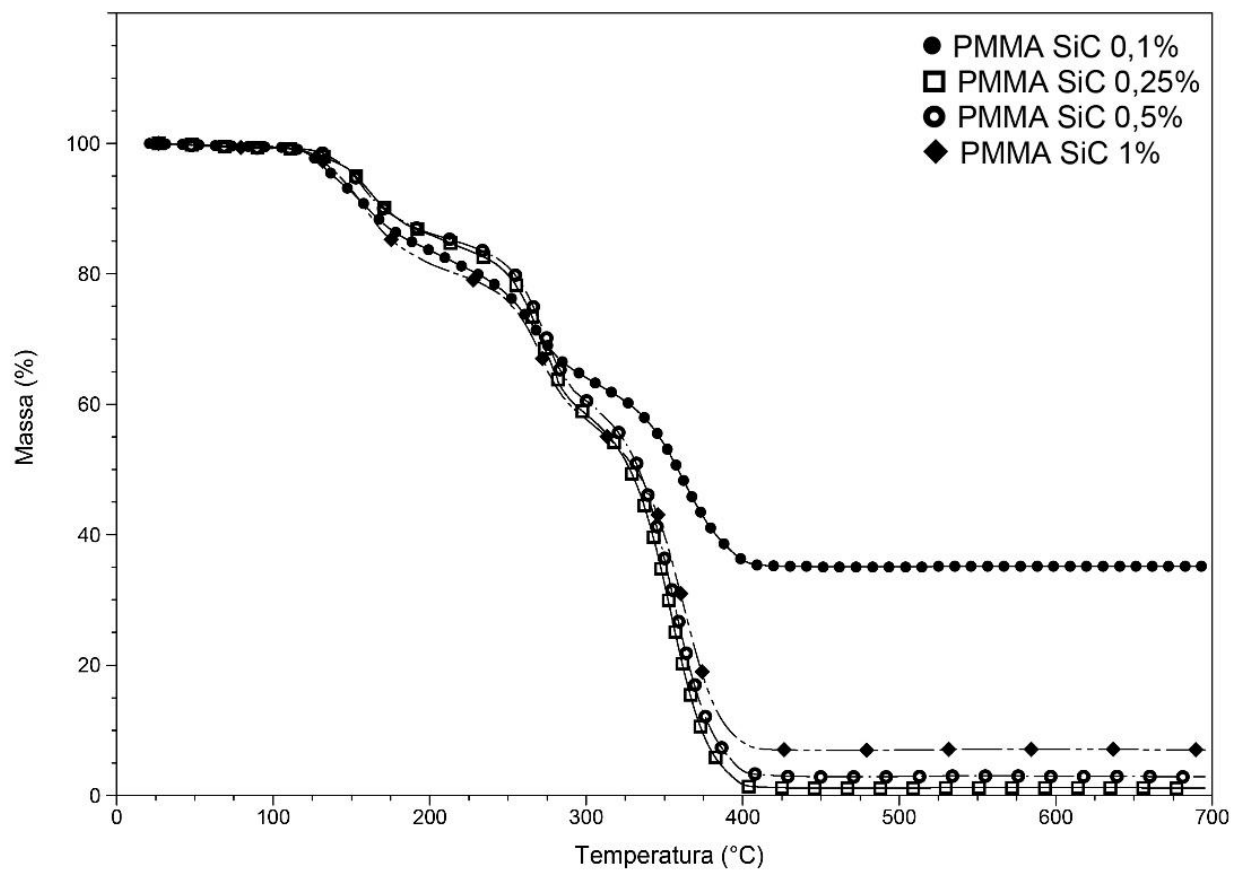

Figura 2. Análises de TGA com as curvas correspondentes aos nanocompósitos sintetizados com $0,1 \% ; 0,25 \% ; 0,5 \%$ e $1 \%$, respectivamente.

Através dos resultados obtidos pela análise de TGA pode- se afirmar que a nanocarga influencia na diminuição da estabilidade térmica dos compósitos.

Tabela 2: Temperaturas de máxima degradação e resíduos dos materiais sintetizados, respectivamente

\begin{tabular}{lllll}
\hline Nanocompósito & $\mathbf{T}_{1 \max }{ }^{\circ} \mathbf{C}$ & $\mathbf{T}_{2 \max }{ }^{\circ} \mathbf{C}$ & $\mathbf{T}_{3 \max }{ }^{\circ} \mathbf{C}$ & Resíduo \\
\hline PMMA puro & 174 & 274 & 355 & 0,30 \\
\hline PMMAISiC 0,1\% & 157 & 269 & 362 & 35,15 \\
\hline PMMAISiC 0,25\% & 164 & 270 & 355 & 1,12 \\
\hline PMMAISiC 0,5\% & 157 & 276 & 353 & 2,85 \\
\hline PMMAISiC 1\% & 158 & 274 & 362 & 6.98 \\
\hline
\end{tabular}

Segundo as imagens das nanopartículas de SiC geradas pela análise de microscopia eletrônica de varredura (FEGSEM) foi possível verificar a morfologia lamelar das partículas de carbeto de silício, dispostas em aglomerados com microestruturas poliédricas, cujas dimensões das partículas são aglomeradas são nanométricas (Figura 3a e 3b).

As imagens de microscopia obtidas dos nanocompósitos possibilitou visualizar a dispersão não homogênea das nanopartículas de SiC pela matriz de PMMA, as quais demostraram a tendência a formação de aglomerados de partículas sob formato de circunferências. 
de energia maiores do que $2 \mathrm{kV}$ para observação, o que restringiu a detecção do elementos químicos presentes nas amostras quantidade mínima de energia pelo equipamento utilizado.
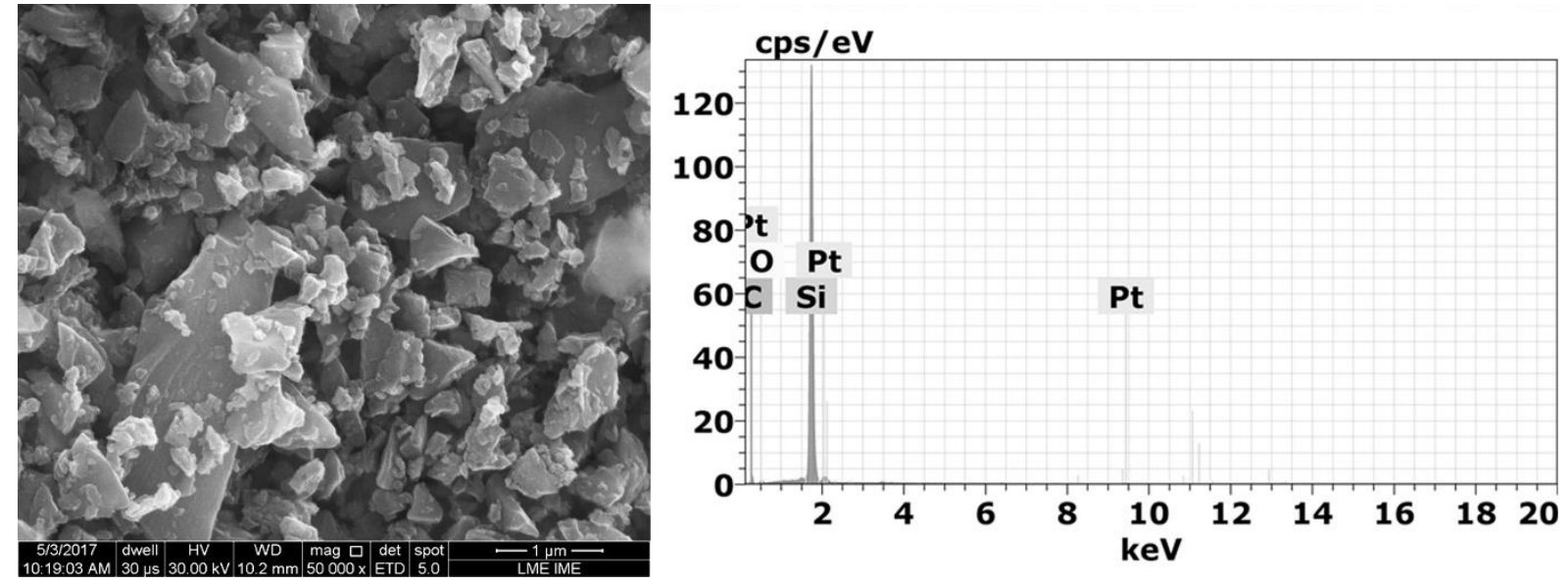

Figura 5. Análise de EDS correspondente a amostra de carbeto de silício, com

Assim como esperado, a análise de EDS do SiC apresentou no espectro das nanocargas, a presença do carbono $(\mathrm{C})$ e silício (Si), comprovando a sua natureza, e o pico observado de platina foi devido ao recobrimento da amostra para análise e observação.

\section{CONCLUSÃO}

Os resultados obtidos pelas técnicas apresentadas e discutidas na pesquisa deixam evidentes o sucesso na produção do filme com matriz de PMMA por polimerização em massa, e mostraram a incorporação de carbeto de silício pelas matrizes poliméricas de PMMA, porém a partir das análises pode sugerir que melhores resultados poderiam ser adquiridos pela organomodificação das superfícies das nanocargas para melhor adesão interfacial, fase orgânica e inorgânica. Os resultados de FTIR e MEV indicam que as nanopartículas de SiC não apresentaram interação interfacial significativa com as matrizes poliméricas produzidas in situ, o que pode ser contornado através da organofilização da superfície da carga. De forma geral, o acréscimo de nanocargas à matriz diminui a estabilidade térmica do polímero.

\section{Agradecimentos}

Nós agradecemos a Coordenação de Aperfeiçoamento de Pessoal de Nível Superior (CAPES), ao Instituto de Macromoléculas Professora Eloisa Mano (IMA) e Instituto Militar de Engenharia (IME) pelo suporte nesse trabalho.

\section{REFERÊNCIAS}

1 Araújo, Rafael S. ; Rezende, Claudinei C. ; Marques, Maria F. V ; Ferreira, Leonardo C. ; Russo, Pietro ; Emanuela Errico, Maria ; Avolio, Roberto ; Avella, Maurizio ; Gentile, Gennaro., Journal of Applied Polymer Science, v. 134, p. 1-10, 2017.

2 A. El-Zaher, Nabawia.; Melegy, Mohamed S.; Guirguis, Osiris W., Natural Science, v.6, p. 859-870, 2014. 
3 Ozkaraoglu, E., Tunc, I. and Suzer, S. N. A. El-Zaher et al. (2009) Polymer, 50, 462466.

$4 \quad$ Khanna, P.K., Singh, N. and Charan, S. (2007). Materials Letters, 61, 47254730.

5 Matsuyama, K. and Mishima, K. (2009). Journal of Supercritical Fluids, 9, 256-264.

6 S Sathish, B Shandar SHekar, Indian Journal of pure and applied physics, vol 52, 2014, 64-67.

7 JI Xiaoli, HAO Hui, WU Qide, ZHOU Bo, SUN Feng. Journal of Wuhan University of Technology-Mater, v22, n.4, 2007.

8 Araujo, S. C.; Kawano, Y. Polímeros: Ciência e Tecnologia, v. 11, n 4, p. 213-221, 2001.

9 Kashiwagi T, Inaba A, Brown JE, Hatada K, Kitayama T, Masuda E. Macromolecules 1986; 19: 2160-2168.

10 Holland BJ, Hay JN. The effect of polymerization conditions on the kinetics and mechanisms of thermal degradation of PMMA. Polym. Degrad.Stab. 2002; 77: 435-439 